\title{
A NOTE ON EIGENVALUES OF NORMAL TRANSFORMATIONS
}

\author{
R. E. L. TURNER ${ }^{1}$
}

There is a substantial literature on variational principles and estimates for eigenvalues of selfadjoint transformations in inner product spaces, most of which depends upon the fact that the associated quadratic forms are real and that the real numbers are totally ordered. Here we show that by using a partial ordering in the complex numbers, defined by means of a complex cone, variational principles and estimates can be obtained for certain classes of normal transformations.

We will assume in what follows that $\mathfrak{X}$ is a finite-dimensional inner product space over the complex numbers, with $(\cdot, \cdot)$ denoting the inner product, and that $N$ is a normal transformation in $\mathfrak{X}$, i.e. $N N^{*}=N^{*} N$. The extensions of the results below to an infinitedimensional Hilbert space are straightforward. We will assume that $N$ has eigenvalues $\lambda_{1}, \lambda_{2}, \cdots, \lambda_{l}$ (with $\operatorname{Re} \lambda_{i+1} \leqq \operatorname{Re} \lambda_{i}$ ) counting multiplicity, and associated orthogonal eigenvectors $w_{i}$. Then $N$ can be written as $N=\sum \lambda_{i} E_{i}$, with $E_{i}$ the orthogonal projection on the span of $w_{i}$. Let $\mu_{1}, \mu_{2}, \cdots, \mu_{k}$ (with $\operatorname{Re} \mu_{i+1} \leqq \operatorname{Re} \mu_{i}$ ) be the eigenvalues, not counting multiplicity. We will assume that

$$
\max _{i<j} 2\left|\arg \left(\mu_{i}-\mu_{j}\right)\right| \leqq \theta<\pi
$$

This would be the case, for example, if $N$ had the form $A+B$, with $A$ selfadjoint, and the norm of $B$ was less than the gap between successive simple eigenvalues of $A$. Denoting the complex numbers by $C$, we let $C \equiv C_{\theta}$ be the cone $\{\alpha \in C|| \arg \alpha \mid \leqq \theta / 2\}$ with $0 \in C$. If $\alpha$ and $\beta$ are complex numbers, we write $\alpha \leqq_{\theta} \beta$ if and only if $\beta-\alpha \in C_{\theta}$. Clearly, the relation $\leqq_{\theta}$ partially orders $C$. The expression $[\alpha, \beta]_{\theta}$ will denote the interval $\left\{\gamma \in C \mid \alpha \leqq_{\theta} \gamma \leqq_{\theta} \beta\right\}$ and we denote by $\tau$, the union of all the intervals $\left[\mu_{i+1}, \mu_{i}\right]_{\theta}$; that is, the maximal totally ordered set containing the eigenvalues of $N$. If $S$ is a set of complex numbers and $S_{e}, e \in E$, an indexed collection of such sets, we make the following definitions:

(1) $\max S=\{\alpha \in S \mid(\alpha+C) \cap S=\alpha\}$,

(2) $\min S=\{\alpha \in S \mid(\alpha-C) \cap S=\alpha\}$,

Received by the editors September 12, 1967.

${ }^{1}$ Supported by NSF Grant GP-5574. 
(3) $\max _{e \in E} S_{e}=\max \bigcup_{e \in E} S_{e}$,

(4) $\min _{e \in E} S_{e}=\min \bigcup_{e \in E} S_{e}$,

where the set containing $\alpha$ alone is denoted by $\alpha$.

Theorems 1, 2, and 3 below are analogues of the classical variational principles for eigenvalues associated with Poincaré, Rayleigh, Ritz, Courant, Fischer, and others. If $N$ is selfadjoint, in which case $\theta=0$, the theorems reduce to the classical ones. In the sequel $N(x)$ will denote $(N x, x) /(x, x)$.

THEOREM 1. The eigenvalues of $N$ can be characterized successively as

$$
\lambda_{n}=\max _{\left(x, w_{i}\right)=0} N(x), \quad i=1,2, \cdots, n-1,
$$

the value $\lambda_{n}$ being assumed for an eigenvector $w_{n}$.

Proof. The proof follows easily from the fact that the numerical range of a normal transformation is the convex hull of its eigenvalues.

Theorem 1 suffers, as does the classical theorem, from the fact that one ordinarily cannot determine the exact eigenvectors. However, it readily provides information on the first and last eigenvalues.

Let $\mathcal{E}_{n}$ denote a generic $n$-dimensional subspace of $\mathfrak{X}$.

THEOREM 2. The eigenvalues of $N$ are characterized by

$$
\lambda_{n}=\min _{\varepsilon_{n-1}} \max _{x \perp_{n-1}} N(x) .
$$

Proof. Denote the right-hand side of (1) by $M_{n}$ and the numerical range of $N$ by $W$. First, if $\alpha \in \lambda_{n}-C$, but $\alpha \neq \lambda_{n}$, and $\varepsilon_{n-1}$ is arbitrary, then $\alpha \notin \max N(x)$ for $x \perp \varepsilon_{n-1}$. For $\lambda_{1}, \lambda_{2}, \cdots, \lambda_{n}$ will be in the cone $\alpha+C$, as will the convex hull $H_{n}$ of $\left\{\lambda_{1}, \cdots, \lambda_{n}\right\}$. For any $\varepsilon_{n-1}$, there is always an $x_{0}$ in $\operatorname{sp}\left\{w_{1}, \cdots, w_{n}\right\}$, the linear span of $w_{1}, \cdots, w_{n}$, such that $x_{0}$ is also orthogonal to $\varepsilon_{n-1}$. Thus $N\left(x_{0}\right) \in H_{n}$, which means $\alpha \notin \max N(x)$ for $x \perp \varepsilon_{n-1}$. It follows that $\lambda_{n} \in M_{n}$.

If $\varepsilon_{n-1}=\operatorname{sp}\left\{w_{1}, \cdots, w_{n-1}\right\}$, then for $x \perp \varepsilon_{n-1}, \max N(x)=\lambda_{n}$, so no point in $\lambda_{n}+C$, other than $\lambda_{n}$, can be in $M_{n}$. Let $K$ be the complement of $\left(\lambda_{n}+C\right) \cup\left(\lambda_{n}-C\right)$. Suppose $\alpha$ is in the lower cone of $K$ and on the boundary of $W$. The intersection of the lower cone of $K$ with the boundary of $W$ consists of an open segment $L_{1}$ (possibly of zero length) of the line $L$ joining two eigenvalues $\lambda_{j}$ and $\lambda_{k}$, with $j<n<k$. Thus any number $\alpha$ on $L_{1}$ is the value of $N\left(\tilde{w}_{k}\right)$ for some $\tilde{w}_{k}$ in the span of $w_{j}$ and $w_{k}$. The subspace

$$
\operatorname{sp}\left\{w_{n}, w_{n+1}, w_{k-1}, \tilde{w}_{k}, w_{k+1} \cdots w_{l}\right\}
$$

is $\tilde{\mathcal{E}}_{n-1}^{\perp}$ for some $\tilde{\mathcal{E}}_{n-1}$ and if $E$ is the orthogonal projection on $\tilde{\mathcal{E}}_{n-1}^{\perp}$, 
then $E N E$, restricted to $\tilde{\mathcal{E}}_{n-1}^{\perp}$, is normal, with eigenvalues $\lambda_{n}, \lambda_{n+1}$, $\cdots, \lambda_{k-1}, \alpha, \lambda_{k+1}, \cdots, \lambda_{l}$. One then sees that the segment from $\lambda_{n}$ to $\alpha$ is in $\max N(x)$ for $x \perp \tilde{\varepsilon}_{n-1}$. Thus every point in $W$ and also in the lower cone of $K$ is in $\max N(x), x \perp \mathcal{E}_{n-1}$, for some subspace $\varepsilon_{n-1}$. The upper cone can be treated similarly and it follows that no interior point of $K \cap W$ can be in $M_{n}$. However, each segment of the boundary of $W$ makes an angle with the real axis not exceeding $\theta / 2$, so no point of $K \cap W$ is in $M_{n}$, and $M_{n}$ consists of $\lambda_{n}$ alone.

Similar arguments yield the complementary max-min Theorem.

THEOREM 3. The eigenvalues of $N$ are characterized by

$$
\lambda_{n}=\max _{\varepsilon_{n}} \min _{x \in \varepsilon_{n}} N(x) .
$$

Other results for selfadjoint problems can be extended to the normal case and combined with the variational principle to give estimates of eigenvalues. We describe two such results.

The first is the Krylov-Temple-Weinstein scheme (see [1, p. 91], and [3]) which for a normal transformation takes the following form:

THEOREM 4. If zero is not an eigenvalues of $N$, then for any $w \neq 0$, the sequence

$$
\alpha_{k}=\frac{\left(N^{k+1} w, N^{k} w\right)}{\left(N^{k} w, N^{k} w\right)}, \quad k=1,2, \cdots
$$

converges to an eigenvalue $\lambda_{j}$ of $N$. Further, if

$$
\epsilon_{k}=\frac{\left\|N^{k+1} w\right\|^{2}}{\left\|N^{k} w\right\|^{2}}-\alpha_{k} \bar{\alpha}_{k}
$$

then $\epsilon_{k}$ converges to zero and there is an eigenvalue of $N$ within distance $\left(\epsilon_{k}\right)^{1 / 2}$ of $\alpha_{k}{ }^{2}$ If we assume that $\operatorname{Re} \mu_{i}>0$ and $\left|\mu_{i+1}\right|<\left|\mu_{i}\right|$ for all $i$, then for all sufficiently large $K$, the pie-shaped region $\left(\alpha_{k}+C\right)$ $\cap\left\{\alpha|| \alpha-\alpha_{k} \mid \leqq\left(\epsilon_{k}\right)^{1 / 2}\right\}$ contains an eigenvalue.

Proof. Using the fact that $N^{k}=\sum \lambda_{i}^{k} E_{i}$ it is easy to show that $\alpha_{k}$ converges to an eigenvalue; namely, the eigenvalue of largest modulus for which $w$ has a nonzero projection on the corresponding eigenspace. Similarly, one sees that $\epsilon_{k} \rightarrow 0$ as $k \rightarrow \infty$.

Let $x \neq 0$ be any vector and let

${ }^{2}$ The referee has kindly pointed out that the enclosure of eigenvalues in discs is orginally due to H. Wielandt (cf. [4, Chapter III] for this and other localization theorems). 


$$
\epsilon=\frac{(N x, N x)}{(x, x)}-\eta \bar{\eta}
$$

where $\eta=(N x, x) /(x, x)$.

If we assume there is no eigenvalue within $(\epsilon)^{1 / 2}$ distance of $\eta$, then

$$
(N x-\eta x, N x-\eta x) /(x, x)>\epsilon .
$$

However, the left-hand side of (2) is $\epsilon$ by definition, yielding a contradiction. Thus there is an eigenvalue in the cone $\alpha_{k}+C$ and one in the disc of radius $\left(\epsilon_{k}\right)^{1 / 2}$ at $\alpha_{k}$. For large $k$ these must be one and the same, from which the last assertion of the theorem follows.

Next, we obtain analogues of Kato's [3] bounds on eigenvalues of selfadjoint transformations. We further suppose, at this point, that $\theta<\pi / 3$.

Lemma. Let $w$ be a unit vector in $\mathfrak{X}$ and define

$$
(N w, w)=\eta, \quad(N w, N w)-\eta \bar{\eta}=\epsilon^{2} .
$$

Suppose $\beta$ is in $\tau$ and $\eta \leqq{ }_{\theta} \beta$. Then there is an eigenvalue of $N$ in

$$
\left(\eta-\epsilon^{2} /(\bar{\beta}-\bar{\eta})+C_{3 \theta}\right) \cap\left(\beta-C_{\theta}\right) .
$$

Proof. If the assertion were false then there would be a point $\xi \in \tau$ which was not in $\eta-\epsilon^{2}(\bar{\beta}-\bar{\eta})^{-1}+C_{3 \theta}$ and such that $[\xi, \beta]_{\theta}$ contained no eigenvalue. Then for each $\lambda_{i},\left(\lambda_{i}-\xi\right)\left(\bar{\lambda}_{i}-\bar{\beta}\right)$ would be in $C_{2 \theta}$ as would $\sum_{i=1}^{n}\left(\lambda_{i}-\xi\right)\left(\bar{\lambda}_{i}-\bar{\beta}\right)\left(E_{i} w, w\right)$ since $\left(E_{i} w, w\right) \geqq 0$. However, the sum is equal to

$$
\|N w\|^{2}+\xi(w, N w)-\bar{\beta}(N w, w)+\xi \bar{\beta}=\eta \bar{\eta}+\epsilon^{2}-\xi \bar{\eta}-\bar{\beta} \eta+\epsilon \bar{\beta},
$$

yielding

$$
\xi(\bar{\eta}-\bar{\beta}) \leqq 2 \theta \eta \bar{\eta}+\epsilon^{2}-\beta \bar{\eta} \text { or } \eta-\epsilon^{2} /(\bar{\beta}-\bar{\eta}) \leqq{ }_{3 \theta} \xi,
$$

contrary to hypothesis.

Theorem 5. Suppose that the spectrum of $N$ in the interval $[\alpha, \beta]_{3 \theta}$ consists of at most a simple eigenvalue. Let $w$ be a unit vector and calculate $\eta$ and $\epsilon$ as above. If $\alpha \leqq_{3 \theta} \eta-\epsilon^{2}(\bar{\beta}-\bar{\eta})^{-1}$ and $\eta+\epsilon^{2}(\bar{\eta}-\bar{\alpha})^{-1} \leqq_{3 \theta} \beta$, then the set

$$
S=[\alpha, \beta]_{\theta} \cap\left[\eta-\epsilon^{2} / \bar{\beta}-\bar{\eta}, \eta+\epsilon^{2} / \bar{\eta}-\bar{\alpha}\right]_{3 \theta} \cap \tau
$$

contains an eigenvalue of $N$.

Proof. This follows immediately from the lemma, applied to $(N, \beta)$ and $(-N,-\alpha)$. 


\section{REFERENCES}

1. K. Yosida, Lectures on differential and integral equations, Interscience, New York, 1960.

2. G. Temple, The computation of characteristic numbers and characteristic functions, Proc. London Math. Soc. (2) 29 (1929), 257-280.

3. T. Kato, On the upper and lower bounds of eigenvalues, J. Phys. Soc. Japan 4 (1949), 334-339.

4. M. Marcus and H. Minc, $A$ survey of matrix theory and matrix inequalities, Allyn and Bacon, Boston, Mass., 1964.

UNIVERSITY OF WISCONSIN 